\title{
Permeability of Urea in N-Carboxymethyl Chitosan-Poly (Vynilalcohol) Blend Membranes for Hemodialysis
}

\author{
R. A. Lusiana, D. Siswanta, Mudasir, and T. Hayashita
}

\begin{abstract}
A series of membranes are prepared by solvent drying method, which were composed of N-carboxymehtyl chitosan blended with poly(vinyl alcohol) [N-CMC/PVA]. The N-CMC/PVA membranes showed improved strength properties and permeability for low-molecular weight compounds. This novel membranes exhibited good permeability properties for small molecules also indicated a decrease in protein adsorption on the surface of membrane. The structure and the morphology of the resulting membranes were characterized by FTIR. NMR, elementary analysis and scanning electron microscopy (SEM).
\end{abstract}

Index Terms-Membranes, n-carboxymethyl chitosan, hemodialysis, permeability.

\section{INTRODUCTION}

Urea is used as a marker for the low-molecular mass toxins removed during the hemodialysis proccess received by patients with chronic renal failure. It has been demostrated that a high urea clearence correlates with long-term patient survival [8]. At the present time hemodialysis is the one of the most important clinical application of blood-contacting biomaterials. The central element of the hemodialysis instrument is a semi-permiable membrane which efficiently transports low molecular weight toxin metabolites without affecting plasma protein [2]. It is reported that the surface properties such as the balance of hydrophilicity/hydrophobicity, electrical charge of biomaterials surface, protein adsorption and selectivity active carrier materials surface are the important factors to affect the character of membranes [10]-[13].

Chitosan have been suggested as materials for membrane due to their good solute permeability and mechanical strength Chitosan has the ability to form thin film membranes with strong mechanical properties [2]. However, the existing amine and hydroxyl groups in chitosan are not strong enough for the interaction with target molecule. Furthermore, the use of unmodified chitosan can trigger protein adsorption on the membrane surface. This is because in acidic condition needed to prepare the chitosan membranes, the amine group on the chitosan backbone will be protonated to form positive charge on the surface, which in turn will lead to further interaction with the negative charge of the protein, hence the protein adsorption occurs. Heparin that has negative charges of sulfonate or sulfate groups, has been suggested as an

Manuscript received January 28, 2013; revised May 30, 2013.

R. A. Lusiana, D. Siswanta, and Mudasir are with the Department of Chemistry and Life Science, Universitas Gadjah Mada, Yogyakarta, Indonesia.

T. Hayashita is with the Department of Materials and Science, Faculty of Science and Technology, Sophia University, Tokyo, Japan. effective way to reduce protein adsorption by chitosan [2], [3].

Several routes can be used to change the surface of chitosan, the key purpose of which is to alter the chemical composition and the surface properties of chitosan to suit specific application [3]-[7]. For the purpose of this research, the modification of chitosan was conducted especially to prevent the interaction between protein and the membrane surface, so that the surface no longer exhibits positive charge. This was achieved by substituting amino groups of chitosan with carboxylic groups under homogeneous conditions [1]-[5]. Therefore, in our research, we attempted to prepare hemodialysis membrane using carboxylate substitution on amino groups of chitosan backbone to make $N$-CMC. Due to its ability to swell in water, it is expected that $N$-CMC should be able to form thin film with good mechanical properties, thus making it a good candidate for use as clinical membranes [5]. The material was prepared by carboxylation of the chitosan and the modified material should have carboxymethyl substituent on the amine, producing negatively charged carboxyl groups on the outer surface. Thus, it was also expected that protein adsorption on the membrane surface would be reduced due to electrical repulsion. Moreover, the ability of the resulted carboxyl group to form stronger hydrogen bonds should increase the membrane selectivity towards the target molecule [7].

Potential applications of $N$-CMC include medical purposes, mainly for the controlled release of drugs, the elaboration of orthopaedic and the industrial effluents [1].

The objective of the present work is to study the mechanism of urea transport across $\mathrm{N}$-CMC matrices and to determine the effects of surface modification through grafting reaction upon the permeation characteristics. In order to produce an appropriate proportion of hydrophobic and hydrophilic as well as mechanical properties, $N$-CMC was blended with compatible synthetic polymers poly(vinyl alcohol)(PVA) [10]. Urea was used as the model compounds in the study. Variation of the diffusion coefficient, the partition coefficient, and the swelling ratio were also studied in the present work. The structure, the morphology, and the mechanical properties of membranes were also investigated.

\section{EXPERIMENTAL}

\section{A. Materials}

Chitosan with deacetylation $>85 \%$ was obtained from Seafresh Chitosan Co, Cirebon (Indonesia). Poly(vinyl alcohol) (weight-average molecular weight $=72,000 \mathrm{~g} / \mathrm{mol}$ ) provided by Aldrich Chemical Co. (St. Louis,MO) as the 
membrane matrix. Chloroacetic acid, sodium hydroxide $(\mathrm{NaOH})$, acetic acid $\left(\mathrm{CH}_{3} \mathrm{COOH}\right)$, isopropanol, ethanol were of analytical grade) was used a a grafting medium of the membranestructure; all provided by Merck Co. (Darmstadt, Germany). Urea was purchased from Sigma Chemical Co (USA).

\section{B. Synthesis of $N-(C M C)$}

Purified chitosan (3g) was dispersed in $65 \mathrm{~mL}$ of isopropanol. After 20 minutes of magnetic stirring at room temperature, $20 \mathrm{~mL}$ aqueous $\mathrm{NaOH} 15 \%$ and amount of various ratio mole of amine: monochloroacetic acid/isopropanol solution $(1: 1 \mathrm{~m} / \mathrm{m})$ were added to the suspension. There action proceeded to the desired time at room temperature for $4 \mathrm{~h}$, the solid product was then filtered, suspended in $150 \mathrm{~mL}$ of ethanol and neutralized with glacial acetic acid. The product was extensively washed with $70 \%$ ethanol and dried at room temperature.

\section{Preparation of Membrane Chitosan/PVA and N-CMC/PVA Film}

Chitosan flakes $(1.5 \mathrm{~g})$ were dissolved in $0.1 \mathrm{M}$ aqueous acetic acid solution $(100 \mathrm{~mL}$ at room temperature with stirring for over night). The solution was filtered using filter paper before used. The solution was poured into a Petri dish ( $2.5 \mathrm{~cm}$ in diameter). The solvent was allowed to evaporate in an oven at $60{ }^{\circ} \mathrm{C}$ for 24 hours. The formed membranes were removed from the dish by soaking in $2 \mathrm{wt} \% \mathrm{NaOH}(\mathrm{aq})$, and washed using distilled water to neutralize the base.

\section{Preparation of Membrane N-CMC/PVA Film}

The copolymer were dissolved in $3 \%$ aqeous acetic acid $(100 \mathrm{~mL})$ with continuous stirring for $20 \mathrm{~h} .1 .5 \mathrm{wt}$ \% PVA solutions were prepared by dissolving PVA flakes in $80{ }^{\circ} \mathrm{C}$ distilled water with stirring for 2-4 h. Then the mixture of copolymer and PVA solution were stirred for $24 \mathrm{~h}$ and poured into petri-dish at room temperature, heated at 50-60 ${ }^{\circ} \mathrm{C}$ for $24 \mathrm{~h}$. After heating treatment, the membranes were soaked in $2 \% \mathrm{NaOH}(\mathrm{aq})$. The membrane was removed from plate and extensively washed using distilled water to remove residual sodium hydroxide. 100-250 $\mu \mathrm{m}$.

\section{E. Analytical Characterization}

The physicochemical characterizations of the chitosan, chitosan/PVA and carboxymethyl chitosan/PVA membranes were carried out by using FTIR spectrometry, NMR spectroscopy, and thermal gravimetry (TGA/DTG). The surface morphology of the N-CMC was studied using an envirenmental scanning electron microscope (SEM, model Hitachi U5800).

\section{F. Permeability Test for the Membranes}

A dialysis chamber was used for determining the permeability of membranes to urea, as a fuction of time, at room temperature. The membranes was clamped between the two compartment using multiple supporting and sealing devides. One compartment was filled with buffer phospate solution, $\mathrm{pH} 7.4$ and the other with a mixtures of solutes containing urea $(50 \mathrm{mg} / \mathrm{dL}, \mathrm{Mw}, 60)$ in phosphate buffer, $\mathrm{pH}$ 7.4. The permeability of solutes through the membrane was analyzed with a Shimadzu UV/VIS at intervals of 0-24 h, employing p-dimethylaminobenzaldehyde reagent for urea. The permeability percentages was calculated using the eq. (1)

$$
\text { Percentage permeated at time } t=C t / C o \times 100
$$

Where $C t$ is the concentration of the solute in the receptor cell difused at time $t$; $C o$ is the initial concentration of the solute in the donor cell and $t$ is the time in minutes.

\section{RESUlts AND DisCUSSION}

\section{A. Synthesis of $N-C M C$}

In this study, we synthesized CMC by direct alkylation of chitosan with monochloroacetic acid at $\mathrm{NaOH}$ (aq). $N$-CMC was synthesized by using $\mathrm{NaOH}_{(\mathrm{aq})} 15 \%$ at room temperature (Fig.1). In mild alkaline medium, only the amine group will be initiated and thus only the amine substitution would occur [5]-[9].

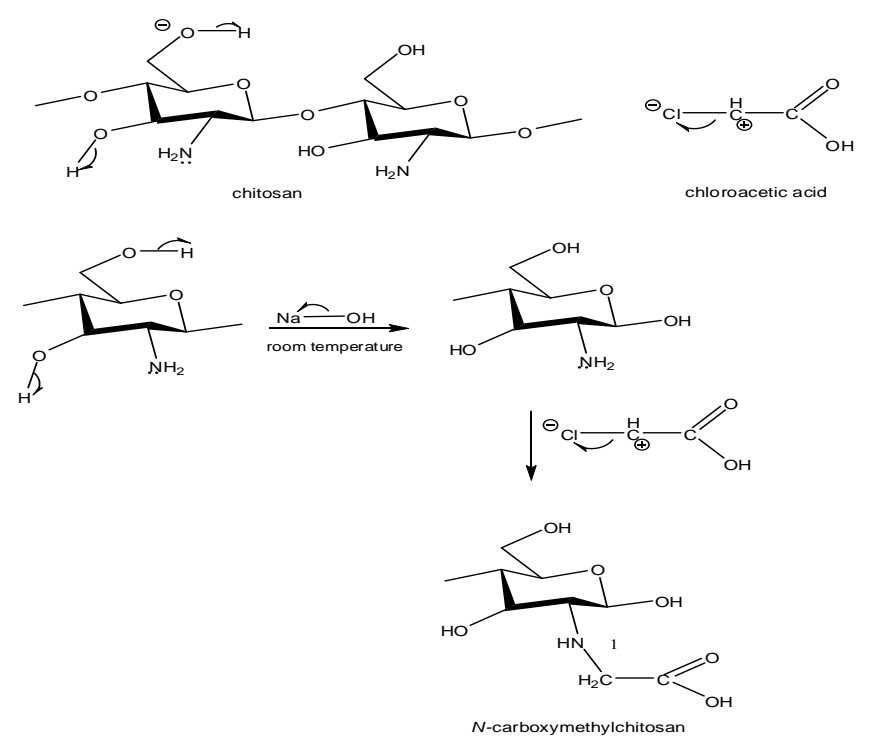

Fig. 1. Scema possible grafted reaction between chitosan with monochloroacetic acid

\section{B. FTIR Spectroscopy}

The structural differences between chitosan and CMC was confirmated by FTIR spectroscopy (Fig.2). The basic characteristic peaks of chitosan were (1) strong and broad band due to the axial stretching of $\mathrm{O}-\mathrm{H}$ and $\mathrm{N}-\mathrm{H}$ bond at $3400 \mathrm{~cm}-1$ (2) splitting band at 1590-1650 cm-1 corresponding toN-H primary bend (3) a band at $1378-1320 \mathrm{~cm}-1$ contribute tothe stretching and bending vibrations of the $\mathrm{C}-\mathrm{N}$ bond of the amide ( 3 band) (4) a band at $900 \mathrm{~cm}-1$ due to C-O stretch..

The spectrum of $\mathrm{N}$-CMC can be shown in Fig.2, the broader band centered at $3400 \mathrm{~cm}-1$ shown the more hydrophilic character of CMC as compared to the chitosan. The strength band at $1600 \mathrm{~cm}-1$ indicating that the most of the primary amine had been changed to secondary amide. Indicating that an $N$-CMC had been prepared. And the strength of the peak at $1411 \mathrm{~cm}-1$ related to the symmetrical vibration of -COO. The peak at $1310 \mathrm{~cm}-1$ indicated to the extension vibration of $\mathrm{C}-\mathrm{O}$ is greatly increased. The strength peak at 1153-897 cm-1 contributes the existence of a C-O-C and $-\mathrm{C}-\mathrm{O}$ bond. The weak peak at $873 \mathrm{~cm}-1$ was 
demonstrating that the carboxymethylation happen at the amino group of chitosan.

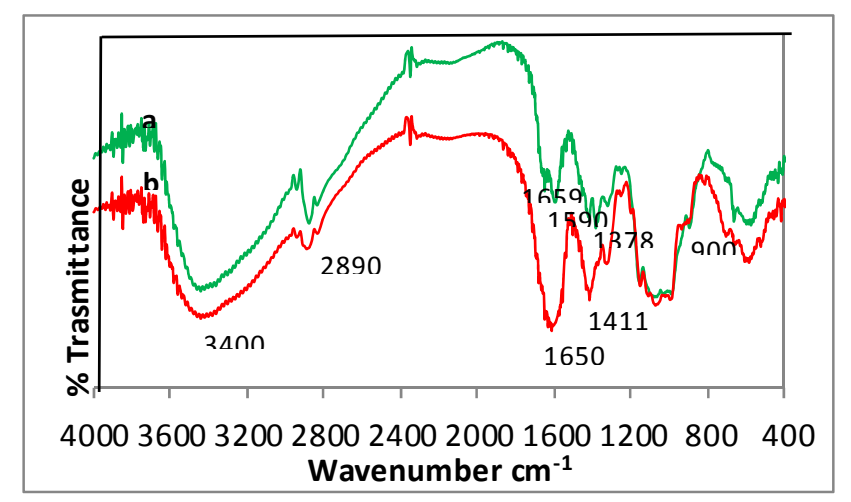

Fig. 2. FT-IR spectrum of (a) chitosan pure, (b) N-carboxymethyl chitosan

\section{C. $H^{l} N M R$}

The H1-NMR spectrum of chitosan (not shown). The signal centerd at $\delta \pm 2.00 \mathrm{ppm}$ was related to the hydrogen atoms of the acetamide groups. The signal detected between 3.10 and $2.90 \mathrm{ppm}$ related to the hydrogen bonded to the $\mathrm{C} 2$ glucosamine rings, while the signal between 3.30 and 4.00 ppm indicated to hydrogen bonded to the carbon atoms $\mathrm{C} 3$, $\mathrm{C} 4, \mathrm{C} 5$ and $\mathrm{C} 6$ of the glucopyranose that are overlap.

The $N$-CMC can be observed by comparing the $\mathrm{H}_{1}$ NMR spectrums of chitosan (Fig. 3). The $\mathrm{H}_{1}$ NMR spectrums at 400 $\mathrm{MHz}$ are informed for $\mathrm{N}-\mathrm{CMC}$ in $\mathrm{D} 2 \mathrm{O} / \mathrm{HCl}$. The existence of $\mathrm{N}$-CMC was demonstrated by the signals in range 3.1-3.4 $\mathrm{ppm}$ in the spectrum, related to mono and disubtitution of the amino groups. The resonance signal of the proton from $\mathrm{N}-\mathrm{CH}_{2} \mathrm{COOH}$ group can be found at $\mathrm{f}(3.22 \mathrm{ppm})$. The results shows that the amino groups were party carboxymethilation along with the amino groups.

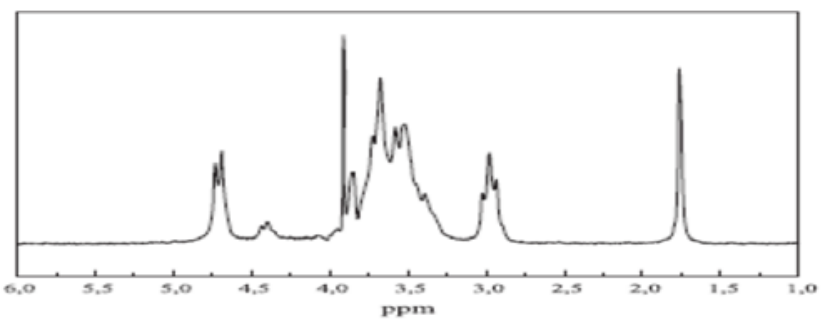

Fig. 3. H-NMR chitosan in D2O/HCl

\section{Water Uptake}

The hydrophilicity of the copolymers is measured by studying their water absorption capacity. It was informed that the chitosan is hydrophobic in nature and its swelling index at $\mathrm{pH} 7,4$ is very low. On grafting the chitosan with carboxyl groups was estimated to increase hydrophilicity, which is confirmed by our results. As shown in Fig. 4 water uptake $\mathrm{N}$-CM C was the higher than chitosan. The higher capacity of $N$-CMC to adsorb water compared to the chitosan are indicated to the higher hydrophilicity of $N$-CMC. The presence of a large number of carboxyl groups in chitosan result in strong hydrogen bonding (may be both intermolecular and intramolecular types), which in turn affect the solubility of CMC in water. Water uptake of sample were decrease by addition of PVA.

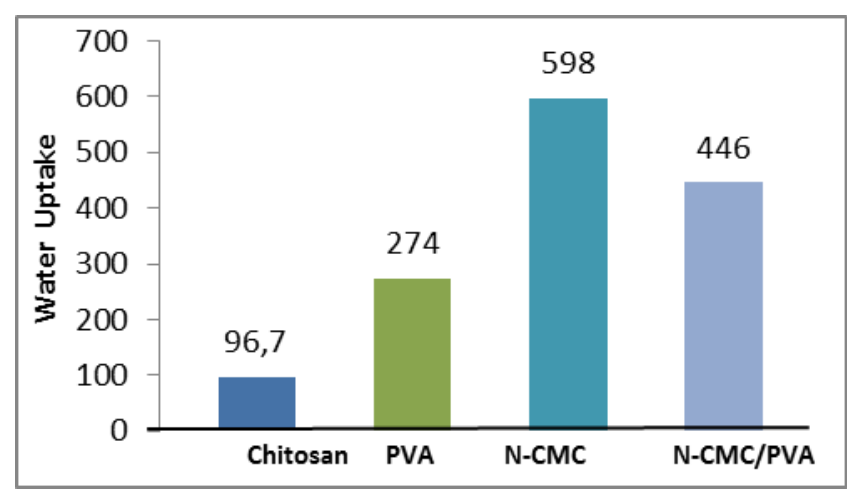

Fig. 4. Water uptake of various membranes

\section{E. Permeation Studies}

The effect of vitamin $\mathrm{B}_{12}$ and albumin in the transport of urea in this research was studied by 3 different feeds content of compounds: 1) feed only contains urea 2) feed containing urea, creatinine and vitamin $\mathrm{B}_{12}$ 3) feed containing urea, creatinine, and albumin vitamin $\mathrm{B}_{12}$. This research used two types of membrane $\mathrm{N}$-CMC with different thicknesses of $0,11 \mathrm{~mm}(\mathrm{M} 1)$ and $0,23 \mathrm{~mm}$ (M2) that can be shown in Figure 5-8. A normal hemodialysis proccess currently takes 5 hour for patients with kidney failure. Therefore, in this research, data collection was carried out for 1-24 h. The permeatibility of urea, creatinine, vitamin $\mathrm{B}_{12}$ and albumin through chitosan, chitosan/PVA and $\mathrm{N}$-CMC membranes were studied in $0,1 \mathrm{M}$ phosphate buffer solution $(\mathrm{pH}$ 7.4) at room temperature.

Urea, due to its lowest molecular weight, has the fastest dialysis rates than does any other metabolite trough all the membranes. Concentrations of the solutes on the acceptor side of the membranes gradually increased and became constant after $10 \mathrm{~h}$. Permeation of urea on the membrane M1 was higher than M2 membrane as shown in Fig. 8. Deppisch et al., (1998) states that a relatively thin membranes can speed up and increase the permeability of the transport proccess. Overall percentage reduction of urea on CMC membrane was much higher than the chitosan membrane. This proved that the role of active groups on the membrane surface was very effective. The active side was actively working on capture of urea on the source phase through the formation of hydrogen bonds.

From Fig. 9 showed, there are no effect of presence vitamin $\mathrm{B}_{12}$ and creatinine on feed solution to urea permeated. However, when the proccess of transport carried out by adding albumin in feed solution, urea permeated being decrease.

\section{F. Surface Morphology of the Film}

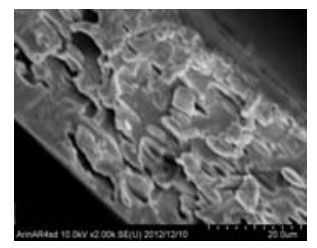

a

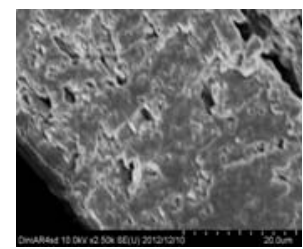

b
Fig. 5. SEM image side surface of N-CMC (a) before used (b) after used 


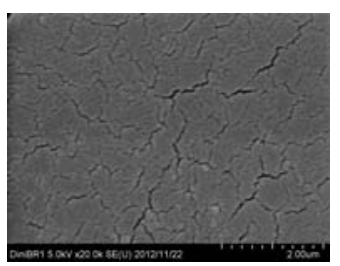

a

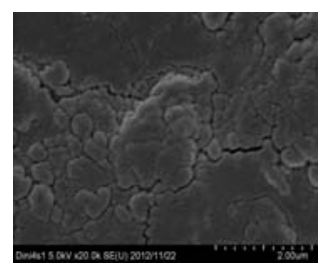

b
Fig. 6. SEM image top surface of N-CMC (a) before used (b) after used

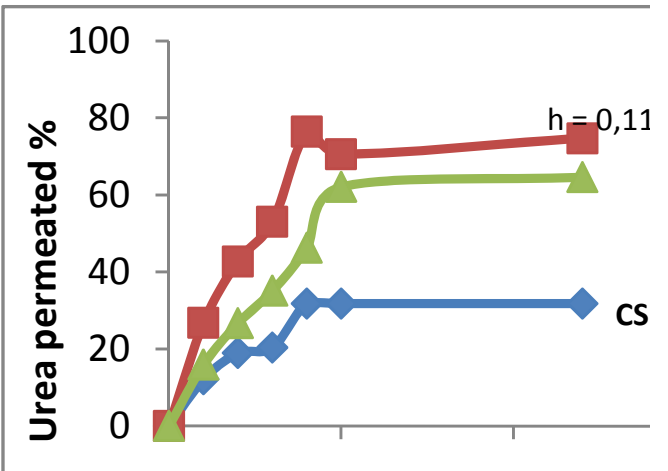

Fig. 7. Urea permeated with time, for 2 thickness of N-CMC membrane and chitosan membranes (feed has urea species

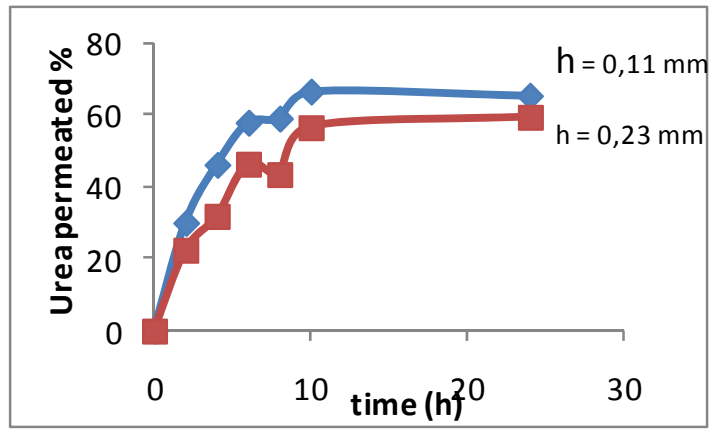

Fig. 8. Urea permeated with time, for 2 thickness of N-CMC membrane (feed has urea, creatinine and cyanocobaltamin species)

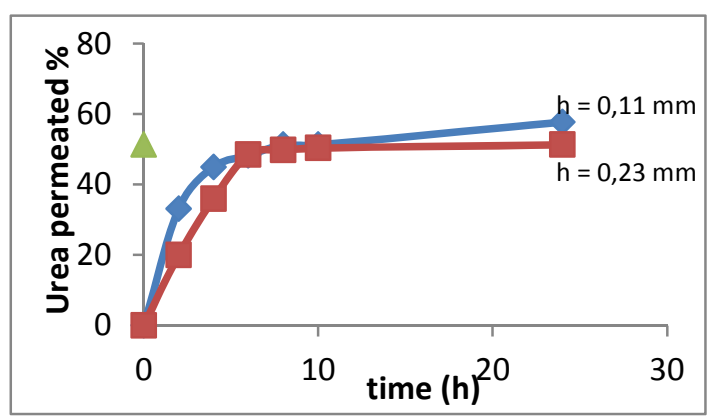

Fig. 9. Urea permeated with time, for 2 thickness of N-CMC membrane (feed has urea, creatinine, cyanocobaltamin and albumin species)

The SEM images at 2000x magnificatian in Fig. 9 show that the N-CMC films have a porous of side surface morphology. The scanning micrograph image of $\mathrm{N}-\mathrm{CMC}$ is shown in Fig. 10. It is no appear that the surface of the membranes covers by albumin.

The image of the SEM of chitosan after used for the transport albumin shows in the Fig. 11. The micrographs clearly shows that surface of chitosan membranes was blocked by albumin. Fouling is probably due to interaction between the positively charge amine groups of chitosan with the negatively charged of protein.

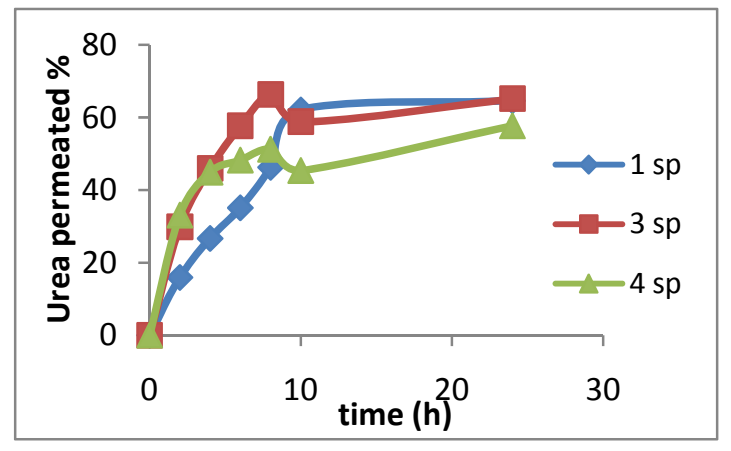

Fig. 10. Urea permeated with time, for thickness $(h=0.11 \mathrm{~mm})$ of N-CMC membrane in different feed contain species
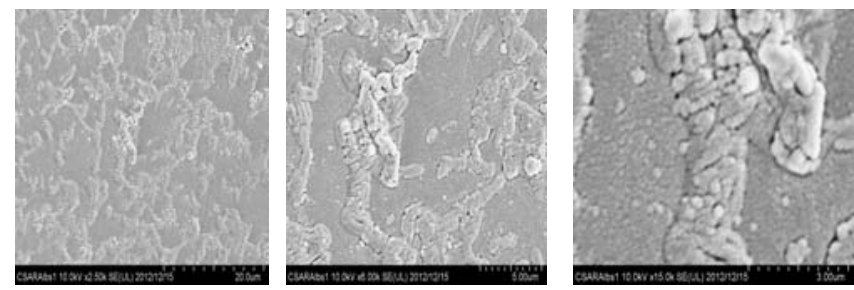

Fig. 11. Scanning electron micrograph of chitosan after used to transport of albumin (a) magnification $2500 x$ (b) $5000 x$ (c) $10000 x$

\section{CONCLUSION}

Biocompatible and mechanically stable membranes could be synthesized from chitosan-g-chloroasetat polymer. The permeability of the membranes for the low moleculat weight solutes like urea was found to be superior to chitosan film. The negative charge of carboxyl groupsis expected to be able to reduce the adsorption of proteins on the membranes surface. In addition, the ability of the carboxyl group to form hydrogen bonds would increase the membranes selectivity towards the target molecule.

\section{REFERENCES}

[1] F. R. Abreu and C. S. P. Filho, "Preparation and Characterization of Carboxymethylchitosan," Polímeros: Ciência e Tecnologia, vol. 15, no. 2, pp. 79-83, 2005.

[2] M. M. Amiji, "Permeability and blood compatibility properties of chitosan-poly (ethylene oxide) blend membranes for haemodialysis," Biomater, vol. 16, pp. 593-599, 1995.

[3] J. Barzin, S. S. Madaeni, and S. Pourmoghadasi, "Hemodialysis Membranes Prepared from Poly (vinyl alcohol): Effect of the Preparation Condotoins on the Morphology and Performance," J. Appl. Polym. Sci., vol. 104, pp. 2490-2497, 2006.

[4] T. Chandy and C. P. Sharma, "Prostaglandin E1-Immobilized Poly (Vinyl Alcohol)-Blended Chitosan Membranes: Blood Compability and Permeability Properties," J. Appl. Polym. Sci., vol. 44, pp. 2145-2156, 1992.

[5] L. Chen, Y. Du, and X. Zeng, "Chemical characteristic of $\mathrm{N}$-carboxymethyl chitosans related to the preparation condition," Carbohyd. Res., vol. 353, pp. 355-359, 2003.

[6] R. Deppisch, M. Storr, R. Buck, and H. Ghl, "Blood material interctions at the surfaces of membranes in medical applicstions," Sep. Purif. Technol., vol. 14, pp. 241-254, 1998.

[7] V. P. Hoven, V. Tangpasuthadol, Y. Angkitpaiboon, N. Vallap, and S. Kiatkanjornwong, "Surface-charged shitosan: Preparation and protein adsorption," Carbohydr. Polym., vol. 68, pp. 44-53, 2007.

[8] S. W. Lewis, P. S. Francis, K. F. Lim, and G. E. Jenkins, "Monitoring urea levels during haemodialysis with a pulsed-flow chemiluminescence analyser," Anal. Chim. Acta, vol. 461, pp. 131-139, 2002.

[9] V. K. Mourya, N. N. Inamdar, and A. Tiwari, "Carboxymethyl chitosan and its applications," Adv. Mat. Lett., vol. 1, pp. 11-33, 2010. 
[10] S. Nakatsuka and A. L. Andrady, "Permeability of Vitamin B-12 in Chitosan Membranes. Effect od Crosslinking and Blending with Poly(vinyl alcohol) on Permeability," J. Appl. Polym. Sci., vol. 44, pp. 17-28, 1992.

[11] N. F. M. Nasir, N. M. Zain, M. G. Raha, and N. A. Kadri, "Characterization of Chitosan-poly (ethylene Oxide) Blends as Haemodialysis Membrane, Am.” J. Sci., vol. 2, no. 12, pp. 1578-1583, 2005.

[12] C. Radhakumary, P. Nair, C. P. Nair, and S. Mathew, "Chitosan-Graft-Poly (vynil acetate) for Hemodialysis Application," $J$ Appl. Polym. Sci., vol. 125, pp. 2022-2033, 2011.
[13] X. Zhao, K. Kato, Y. Fukumoto, and K. Nakamae, Int. J. Adhes. and Adhesiv., vol. 21, no. 227, 2001.

R. A. Lusiana was born in Grobogan, Central Java (Indonesia) in Dec 2 rd 1970. She received her master's degree in Analytical Chemistry from the University Gadjah Mada, Indonesia. At present, she is doing $\mathrm{PhD}$ in Department Chemistry, FMIPA, Universitas Gadjah Mada under the supervision of Dr. Dwi Siswanta, Prof. Dr. Mudasir. Her area of interest in membrane analyzer. 